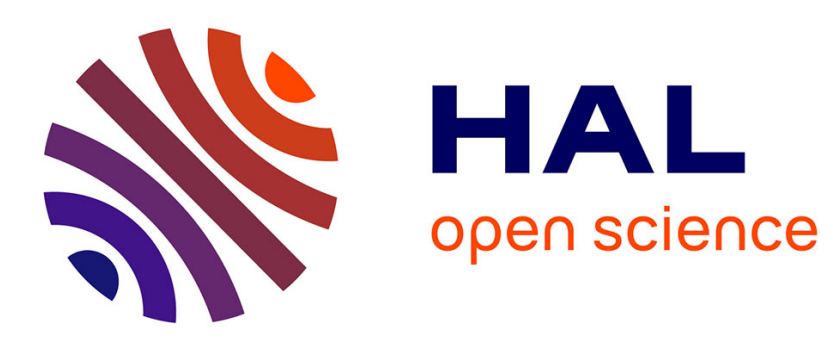

\title{
Modelling a ratchet with cold atoms in an optical lattice
}

Cécile Robilliard, D. Lucas, G. Grynberg

\section{To cite this version:}

Cécile Robilliard, D. Lucas, G. Grynberg. Modelling a ratchet with cold atoms in an optical lattice. Applied physics. A, Materials science \& processing, 2002, 75 (2), pp.213-216. 10.1007/s003390201333. hal-00003467

\section{HAL Id: hal-00003467 \\ https://hal.science/hal-00003467}

Submitted on 3 Dec 2004

HAL is a multi-disciplinary open access archive for the deposit and dissemination of scientific research documents, whether they are published or not. The documents may come from teaching and research institutions in France or abroad, or from public or private research centers.
L'archive ouverte pluridisciplinaire HAL, est destinée au dépôt et à la diffusion de documents scientifiques de niveau recherche, publiés ou non, émanant des établissements d'enseignement et de recherche français ou étrangers, des laboratoires publics ou privés. 


\title{
Modelling a ratchet with cold atoms in an optical lattice
}

\author{
C. Robilliard*, D. Lucas ${ }^{\dagger}$, and G. Grynberg \\ Laboratoire Kastler Brossel, \\ Département de Physique de l'Ecole Normale Supérieure \\ 24, rue Lhomond, F-75231 Paris cedex 05, France
}

\begin{abstract}
In this paper, we present an experimental study of a model ratchet consisting of lasercooled rubidium atoms localized in an asymmetric periodic potential. This potential is obtained from a near-resonant light field and an additional static magnetic field. We show that there is a net motion of atoms with a mean velocity on the order of a few $\mathrm{cm} / \mathrm{s}$ and we investigate the role of the asymmetry of the potential and of the dissipation in the atomic motion. A link with the stochastic resonance is mentioned.

PACS: 32.80.Pj, 32.60.+i, 87.10.+e, 05.40.+j, 05.60.+w
\end{abstract}

\section{INTRODUCTION}

Due to its application to molecular motors that convert chemical energy into mechanical energy, for example in muscles, the physics of ratchets has been increasingly studied in recent years [1]. From a general point of view, a ratchet is a non-linear system which is able to convert unbiased non-equilibrium fluctuations into mechanical work. A common model for ratchets consists of Brownian particles evolving in a periodic but spatially asymmetric potential in the presence of dissipation. Because both parity and time reversal symmetry are violated, a directional motion of particles is possible although there is apparently no net force acting on them [2].

More elaborate theoretical models consider the particles to exist in two states each of them experiencing a different potential. Both potentials are periodic but asymmetric. When the transition rates between the two states differ from their values at Boltzmann equilibrium, a directional average motion is predicted [3]. This paper presents an experimental realisation of a very similar model, using laser-cooled rubidium atoms.

In section II we explain how to obtain a ratchet-like system for cold atoms. The experimental set-up is presented in section III, and the next two sections are devoted to the experimental study of the influence of spatial asymmetry (section IV) and dissipation (section V), respectively. The role of dissipation here is very similar to the one found in stochastic resonance $[4,5]$.

\section{MODELLING A RATCHET WITH COLD ATOMS}

Atoms cooled and manipulated by light provide a very versatile medium to model many phenomena usually observed in other systems. In the present case, we combine a near-resonant light field with a static magnetic field to tailor a potential which is periodic but asymmetric. In this system, dissipation originates from optical pumping between different Zeeman sublevels.

We first consider the interaction of an atom with an electromagnetic field, the atom having an electronic transition from a ground state with an angular momentum $F=1$ to an excited state with the same angular momentum $F^{\prime}=1$. If the electromagnetic field is polarized in the $x O y$ plane (no $\pi$ component), it is well-known that all the physics can be described in terms of a $\Lambda$ system which includes the two ground state Zeeman sublevels $m=-1$ and $m=1$ and the excited sublevel $m^{\prime}=0[6]$. This transition has been widely studied in the context of velocity selective cooling by population trapping (VSCPT) [7]. Furthermore, if the light intensity is sufficiently small or if the frequency detuning $\Delta$ from the atomic resonance is sufficiently large, it is possible to eliminate adiabatically the excited substate and to describe all the physical processes inside the two-level subspace $\{m=-1, m=1\}$ [6]. The master equation then contains (i) a Hamiltonian evolution in the optical bipotential due to the light-shifts, (ii) damping processes originating from absorption-spontaneous emission cycles. Because such a $\Lambda$ system accommodates a state $|N C\rangle$ which is not coupled to the excited state [8], the corresponding optical potential $V_{N C}$ is flat $\left(V_{N C}=0\right)$ and the

*Permanent address: Laboratoire Collisions, Agrégats, Réactivité (UMR 5589, CNRS and Université Paul Sabatier), Toulouse, France.

${ }^{\dagger}$ Present address: Clarendon Laboratory, Parks Road, Oxford OX1 3PU, UK. 
Hamiltonian appears as the sum of the kinetic energy $p^{2} / 2 M$ (where $M$ is the atomic mass and $p$ the momentum) and of the potential energy $V_{C}|C\rangle\langle C|$ (with $\langle N C \mid C\rangle=0$ ).

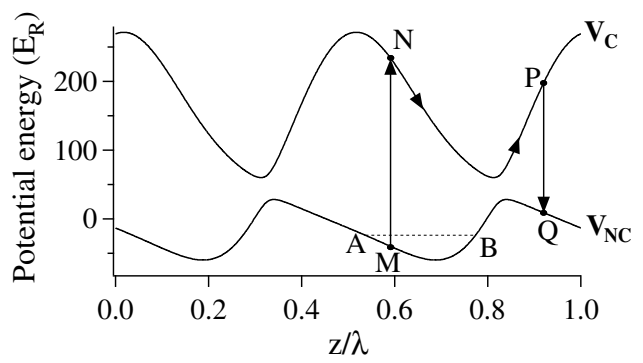

FIG. 1. Optical bipotential in the "lin $\theta$ lin" configuration with a longitudinal magnetic field $\mathbf{B}_{0}$. The parameters are $\theta=45^{\circ}$, $\Delta / \Gamma=2, \Omega / \Gamma \simeq 0.65$ and $\Omega_{0}=60 \omega_{R}$ (where $E_{R}=\hbar \omega_{R}=\hbar^{2} \omega^{2} /\left(2 M c^{2}\right)$ is the recoil energy). The curves represent the exact optical potential, obtained by diagonalizing the total Hamiltonian (light-shift and Zeeman-shift). An atom oscillating between $\mathrm{A}$ and $\mathrm{B}$ can shift to the neighbouring well because of a transition in $V_{C}$.

In the following, we consider a unidimensional (1D) situation with two counterpropagating light beams $\mathbf{E}_{1}$ and $\mathbf{E}_{2}$ of the same amplitude $E_{0}$, the same frequency $\omega=c k$ and linear polarizations $\mathbf{e}_{1}=\cos (\theta / 2) \mathbf{e}_{x}+\sin (\theta / 2) \mathbf{e}_{y}$ and $\mathbf{e}_{2}=$ $\cos (\theta / 2) \mathbf{e}_{x}-\sin (\theta / 2) \mathbf{e}_{y}$ ("lin $\theta$ lin" configuration). The optical potential associated with $|C\rangle$ is $V_{C}=\hbar \Delta s D(z) / 2$ where $\Delta=\omega-\omega_{0}$ ( $\omega_{0}$ : atomic resonance frequency), $s=\frac{\Omega^{2} / 2}{\Delta^{2}+\Gamma^{2} / 4}, \Omega=d E_{0} / \hbar$ ( $d$ being the reduced matrix element of the electronic dipole moment), $\Gamma$ the natural width of the excited state and $D(z)=1+\cos \theta \cos (2 k z)$. In order to have a spatially modulated potential $V_{N C}$, a small magnetic field $\mathbf{B}_{0}$ along $O z$ can be added. The Zeeman shift then leads to

$$
V_{N C}=-\hbar \Omega_{0} \sin \theta \sin (2 k z) / D(z)
$$

with $\hbar \Omega_{0}=g \mu_{B} B_{0}$ ( $g$ : Landé factor of the ground state, $\mu_{B}$ : Bohr magneton). The potentials $V_{N C}$ and $V_{C}$ (the latter now differs slightly from $\hbar \Delta s D(z) / 2$ because of the Zeeman effect) are shown in Fig. 1. In this system, efficient Sisyphus cooling can occur for $\Delta>0[9]$ through the following mechanism: an atom moving in $|N C\rangle$ can be transferred into $|C\rangle$ by motional coupling, which is most efficient near the anti-crossing points of the potential. It then climbs a potential hill of $V_{C}$ before being optically pumped back into $|N C\rangle$. Because the optical pumping rate from $|C\rangle$ to $|N C\rangle$ is maximum near the top of the potential hill, the kinetic energy of the atom decreases. This process finally leads to cooling and trapping of a large fraction of the atoms in the asymmetric wells of the uncoupled potential. Such a system is called grey lattice, and the required light beams $\mathbf{E}_{1}$ and $\mathbf{E}_{2}$ are referred to as "grey lattice beams" in the following.

What happens to an atom oscillating in such a well (between A and B in Fig. 1) ? The transition probability from $|N C\rangle$ to $|C\rangle$ due to $B_{0}$ is maximum near A. Once in $|C\rangle(\mathrm{M} \rightarrow \mathrm{N}$ in Fig. 1), the atom undergoes an oscillation that is interrupted by a spontaneous Raman process that brings the atom back into $|N C\rangle(\mathrm{P} \rightarrow \mathrm{Q}$ in Fig. 1). The jumps between the potential curves thus tend to drag the atom to the right-hand side of Fig. 1. Note that because of the long lifetime of atoms in $|N C\rangle$, a jump from $|N C\rangle$ to $|C\rangle$ is a rather seldom event, so that the atoms undergo many oscillations in a well of the $|N C\rangle$ potential between two jumps ${ }^{1}$. One recognizes a mechanism similar to the stochastic resonance, where synchronisation of the noise (here optical pumping) induces the motion [5].

Inspecting Eq. 1, three basic properties of the potential $V_{N C}$ can be noticed: (i) $V_{N C}$ changes sign when $\mathbf{B}_{0}$ is reversed; (ii) $V_{N C}$ has opposite asymmetries for $\theta=\theta_{0}$ and $\theta=-\theta_{0}$; (iii) $V_{N C}$ is symmetrical for $\theta=0$, $\pm \pi / 2$. The experimental results are in good agreement with these features of the potential (see [10] and section IV).

\section{EXPERIMENTAL SET-UP}

To perform the experimental study, we start from rubidium atoms $\left({ }^{87} \mathrm{Rb}\right)$ trapped and cooled in a magneto-optical trap (MOT) [11]. At a certain time we switch off the MOT beams and inhomogeneous magnetic field and switch on

\footnotetext{
${ }^{1}$ There is another cause to the jumps which is the non-adiabatic coupling. This coupling is essential for the velocity damping.
} 
the grey lattice beams operating on the blue side of the $5 S_{\frac{1}{2}}(F=1) \rightarrow 5 P_{\frac{3}{2}}\left(F^{\prime}=1\right)$ transition as well as repumping beams detuned by $2 \Gamma$ on the blue side of the $5 S_{\frac{1}{2}}(F=2) \rightarrow 5 P_{\frac{3}{2}}\left(F^{\prime}=2\right)$ transition (to avoid a leakage of atoms into the $F=2$ hyperfine sublevel) and the static magnetic field $\mathbf{B}_{0}$. The counterpropagating grey lattice beams are vertical while the repumping beams lie in the horizontal plane. This arrangement allows a partial compensation of the effect of gravity while ensuring that the repumping beams do not contribute to the vertical motion.

The spatial displacement of cold atoms is measured using a CCD camera by direct imagery of the atomic cloud (whose initial radius is $\sim 1 \mathrm{~mm}$ ). One technique is to use the faint image of the grey lattice itself. This requires the averaging of a significant number of images $(\sim 100)$ because atoms in $|N C\rangle$ scatter very few photons. An example of measurements done with this method is shown in [10]. Instead of tracking the faint image of the grey lattice, one can also apply after a grey lattice phase $\tau_{L}$ a flash of two beams nearly resonant on the $5 S_{\frac{1}{2}}(F=1) \rightarrow 5 P_{\frac{3}{2}}\left(F^{\prime}=2\right)$ and $5 S_{\frac{1}{2}}(F=2) \rightarrow 5 P_{\frac{3}{2}}\left(F^{\prime}=3\right)$ transitions. The results presented hereafter were obtained with this technique, which is easier and quicker as the atoms scatter many photons.

In fact, we can get more precise information than the average velocity. We can determine the whole velocity distribution using a ballistic method. For this purpose, we create a sheet of light $10 \mathrm{~cm}$ below the cloud of cold atoms. This sheet of light is resonant on the $5 S_{\frac{1}{2}}(F=1) \rightarrow 5 P_{\frac{3}{2}}\left(F^{\prime}=2\right)$ transition. When the grey lattice beams and $\mathbf{B}_{0}$ are switched off, the rubidium atoms fall freely. The time-variation of the absorption when the atoms cross the sheet of light is directly related to the velocity distribution [11]. Such distributions are presented in [10].

\section{THE ROLE OF SPATIAL ASYMMETRY}

One of the two essential features of a ratchet is the spatial asymmetry of the potential. In our system, we can easily modify its shape by changing the angle $\theta$ between the polarisations of the counterpropagating waves or changing the magnetic field.

We thus measured the displacement $d$ of the atomic cloud between $t=0$ and $t=\tau_{L}$. In fact, to avoid the supplementary effect of gravity, we measured the displacements $d_{+}$and $d_{-}$for opposite values of $\mathbf{B}_{0}$ and plotted the variation of $\bar{d}=\left(d_{-}-d_{+}\right) / 2$. We show in Fig. 2.a the dependence of $\bar{d}$ versus $\theta$ for $\Omega_{0}=-170 \omega_{R}, \Delta / \Gamma=2, s \simeq 0.2$ and $\tau_{L}=6 \mathrm{~ms}$. As expected, $\bar{d}=0$ when the potentials $V_{C}$ and $V_{N C}$ become symmetric, i.e. for $\theta=0, \pm \pi / 2$. We also find that $\bar{d}$ is an odd function of $\theta$, in agreement with the change of asymmetry of the potentials in the transformation $\theta \rightarrow-\theta$.

We show in Fig. 2.b the variation of the displacement $\bar{d}$ as a function of the Zeeman shift $\Omega_{0}$. At low $\Omega_{0}$, the displacement exhibits a slow increase with $\Omega_{0}$. This is because there are not so many trapped atoms when the potential wells of $V_{N C}$ are too shallow ${ }^{2}$ and because the rate of transitions from $|N C\rangle$ to $|C\rangle$ due to the magnetic coupling is roughly a quadratic function of $\Omega_{0}$. When $\Omega_{0}$ becomes too large, the perturbative expression of $V_{N C}$ (Eq. 1) is no longer valid. A more accurate calculation shows that the potentials lose their asymmetry for high values of $\Omega_{0}$. We find indeed a decrease of $\bar{d}$ when the Zeeman shift reaches a value that becomes comparable with the light-shift $\Delta s / 2$.

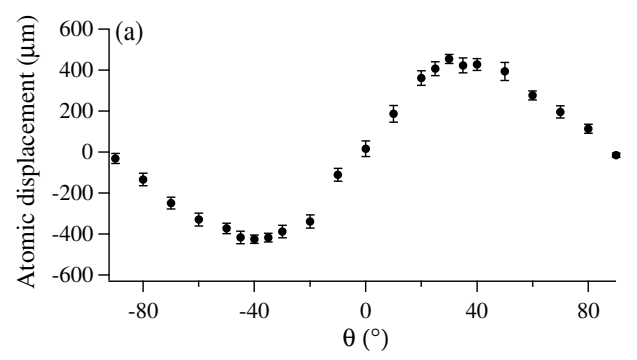

\footnotetext{
${ }^{2}$ Because of the neighbouring transitions $F=1 \rightarrow F^{\prime}=0$ and $F=1 \rightarrow F^{\prime}=2$, the optical potential $V_{N C}$ is not perfectly flat even when $\Omega_{0}=0$. The effect of $\Omega_{0}$ is correctly described only when the Zeeman shift becomes larger than the light-shift due to the remote transitions.
} 


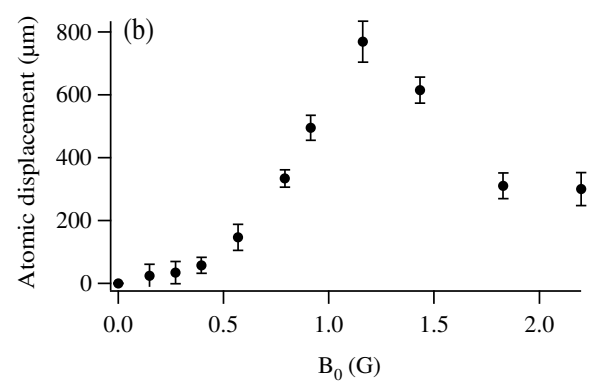

FIG. 2. (a) Atomic displacement $\bar{d}=\left(d_{-}-d_{+}\right) / 2$ as a function of the angle $\theta$ between the linear polarizations of the two lattice beams, for $\Delta / \Gamma=2, \Omega_{0}=-170 \omega_{R}, s \simeq 0.2, \tau_{L}=6 \mathrm{~ms}$. (b) Atomic displacement $\bar{d}=\left(d_{-}-d_{+}\right) / 2$ as a function of the magnetic field amplitude $\mathbf{B}_{0}=\hbar \Omega_{0} /\left(g \mu_{B}\right)$, for $\Delta / \Gamma=2, \theta \simeq 45^{\circ}, s \simeq 0.15, \tau_{L}=6 \mathrm{~ms}$. The largest displacement is obtained for $\left|\Omega_{0}\right| \sim \Delta s / 2$.

\section{THE ROLE OF DISSIPATION}

In this section, we want to focus on the role of dissipation in the net motion of the atoms.

To do this, we measured the atomic displacement for different sets of laser intensity $I$ and detuning $\Delta$ such that only the optical pumping rate varies: if we change $I$ and $\Delta$ proportionally, then the potential curves are not affected since they scale as $\Delta s \propto I / \Delta$, while the optical pumping rate scales as $I / \Delta^{2}$. The resulting curve is shown in Fig. 3: we find that the net atomic flux increases with dissipation, which is indeed expected in the oscillating regime, where the atom oscillates many times in a given potential well between two otical pumping processes. Such a behaviour is also expected in the framework of stochastic resonance: the displacement grows initially with noise [5] but decreases at large noise amplitude. The shape of the curve is in good agreement with semi-classical Monte-Carlo numerical calculations. Unfortunately, our experimental set-up does not allow us to explore the jumping regime (in this case the process can also be explained in terms of rectified forces [12]).

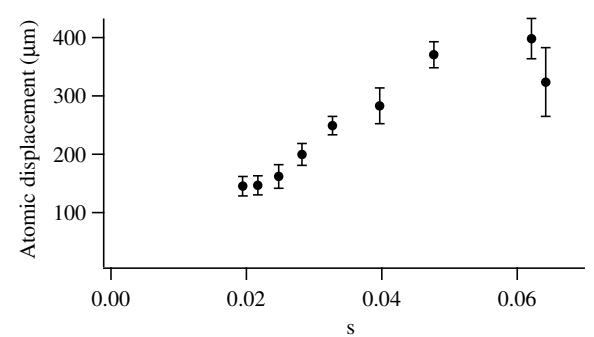

FIG. 3. Measured atomic displacement as a function of the saturation parameter $s$. Both the laser intensity and detuning are changed in order to keep the potentials constant while varying the optical pumping rate. The parameters are $\Omega_{0}=-170 \omega_{R}$, $\theta=45^{\circ}, \Delta s \simeq 150 \omega_{R}, \tau_{L}=6 \mathrm{~ms}$.

We also studied the role of dissipation through semi-classical numerical Monte-Carlo simulations of the atom dynamics [13], the results of which are in qualitative agreement with the experimental observations. For example, we show in Fig. 4 the time evolution of the spatial distribution function of an initially well-localized atomic sample (parameters: $\theta=45^{\circ}, \Delta / \Gamma=2, \Omega / \Gamma=1.8, \Omega_{0}=250 \omega_{R}$ ). The initial sample appears in Fig. 4.a for reference, and we show in Fig. 4.b the spatial distribution of the atoms after $750 \overline{\tau_{C}}$, where $\overline{\tau_{C}}$ is the atomic lifetime in $|C\rangle$ averaged over one spatial period. The directed motion and the spreading of the density are clearly visible in these data. The average velocity is $v=1.5 v_{R}$, which is 3 times smaller than the velocity found experimentally in the same conditions. Several explanations can be given for this discrepancy: first, it is well-known that in the case of a $F=1 \rightarrow F^{\prime}=1$ transition the atom dynamics for small $B_{0}$ requires a full quantum treatment [7] as long as $\Omega_{0}<s \Delta$ [14]. Indeed, there might be some relation between this discrepancy and the observation by Reimann et al. [15] that quantum tunnelling can significantly modify the flux in a similar problem. Furthermore, we neglect the neighbouring hyperfine transitions in the simulation.

If we now force the detailed balance to be verified, the situation is that of an "artificial thermal equilibrium". This can be done easily in the simulation by replacing the actual transition rate $\Gamma_{N C}$ from $|N C\rangle$ to $|C\rangle$ by one which 
is determined from $\Gamma_{C}$ to obey detailed balance: $\Gamma_{N C}(z)=\Gamma_{C}(z) \exp \left[\frac{U_{N C}(z)-U_{C}(z)}{k_{B} T}\right]$ where $T$ corresponds to the experimental temperature. The resulting spatial distribution is represented in Fig. 4.c: the atomic cloud spreads symmetrically and no net flux exists. In this case indeed, the transition rate $\Gamma_{N C}$ is largest on the other side of the well (near B in Fig. 1), which inhibits the directed motion.
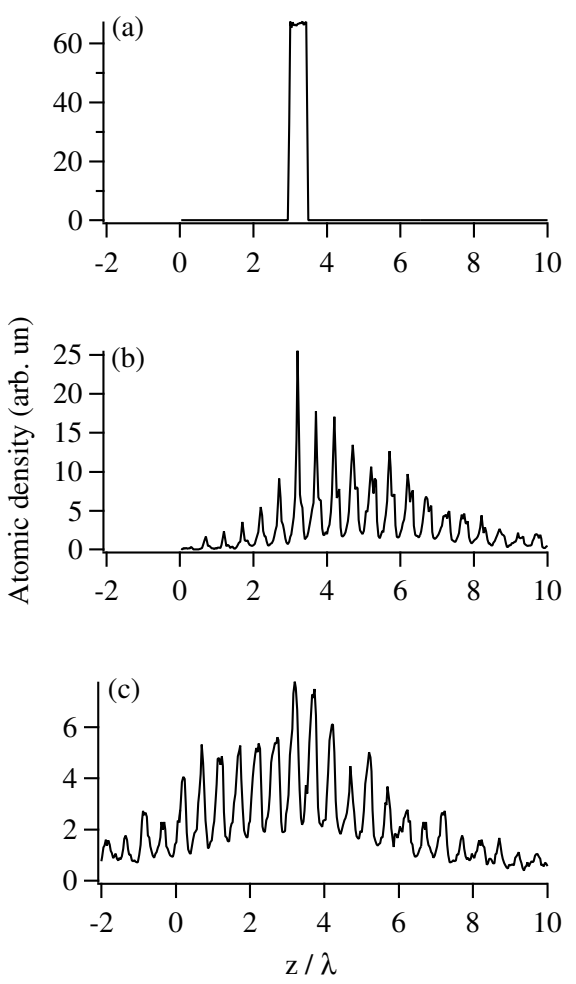

FIG. 4. Numerical results obtained by a semi-classical Monte-Carlo simulation, showing the temporal evolution of the spatial distribution of the atoms, for $\theta=45^{\circ}, \Delta / \Gamma=2, \Omega / \Gamma=1.8, \Omega_{0}=250 \omega_{R}$. (a) Initial spatial distribution. (b) Spatial distribution after $750 \overline{\tau_{C}}$, where $\overline{\tau_{C}}$ is the atomic lifetime in $|C\rangle$ averaged over one spatial period. (c) Spatial distribution after the same time of evolution, when we force the transition rates between the two states to obey detailed balance. Note the symmetry of the distribution.

\section{CONCLUSION}

In this paper, we have used cold atoms in an optical lattice as a model system for a ratchet, which was originally considered in the context of biophysics. The next step to make our system even more versatile would consist of localizing the atoms in a far-off resonance optical lattice where there is virtually no optical pumping. Dissipation could then be added in a fully controlled manner, either with an additional light beam or by modulating for example the intensity of the lattice beams.

Another direction could be to pre-cool the atoms down to the quantum regime, in order to realize a quantum ratchet whose properties are known to be noticeably different from those of a classical ratchet [15].

Finally, the authors wish to thank S. Guibal, C. Jurczak, J. W. Tabosa and F.-R. Carminati for fruitful discussions. This work was partly supported by the European Commission (TMR network "Quantum Structures", contract FRMXCT96-0077) and by the Conseil Régional d'Île-de-France (Convention SESAME n E.1220, "Atomes ultrafroids : vers de nouveaux états de la matière"). Laboratoire Kastler Brossel is an unité mixte de recherche de l'Ecole Normale Supérieure, de l'Université Pierre et Marie Curie et du CNRS. 
[1] For a review of the field, see the other articles in this issue, and references therein.

[2] P. Curie, J. Phys. (Paris) 3, 393 (1894).

[3] J. Prost et al., Phys. Rev. Lett. 72, 2652 (1994); J.-F. Chauwin, A. Ajdari and J. Prost, Europhys. Lett. 27, 421 (1994); F. Jülicher, A. Ajdari and J. Prost, Rev. Mod. Phys. 69, 1269 (1997).

[4] L. Gammaitoni, P. Hänggi, P. Jung and F. Marchesoni, Rev. Mod. Phys. 70, 223 (1998).

[5] L. Sanchez-Palencia, F.-R. Carminati, M. Schiavoni, F. Renzoni and G. Grynberg, to be published.

[6] C. Cohen-Tannoudji, in Fundamental Systems in Quantum Optics, Proceedings of the Les Houches Summer School of Theoretical Physics, Les Houches, 1990, Session LIII, edited by J. Dalibard, J.-M. Raimond and J. Zinn-Justin (Elsevier Science, Amsterdam, 1992); H. Metcalf and P. van der Straten, Phys. Rep. 244, 203-286 (1994).

[7] A. Aspect et al., Phys. Rev. Lett. 61, 826 (1988); B. Saubamea et al., Phys. Rev. Lett. 79, 3146 (1997).

[8] G. Alzetta et al., Nuovo Cimento B 36, 5 (1976); E. Arimondo and G. Orriols, Lett. Nuovo Cimento 17, 333 (1976).

[9] G. Grynberg and J.-Y. Courtois, Europhys. Lett. 27, 41 (1994).

[10] C. Mennerat-Robilliard et al., Phys. Rev. Lett. 82, 851 (1999).

[11] W. D. Phillips, in Proceedings of the International School of Physics Enrico Fermi, Course CXXXI, edited by A. Aspect, W. Barletta and R. Bonifacio (IOS Press, Amsterdam) 1996, p. 209.

[12] R. Grimm et al., JETP Lett. 54, 615 (1991); J. Söding and R. Grimm, Phys. rev. A 50, 2517 (1994).

[13] Y. Castin, J. Dalibard and C. Cohen-Tannoudji, in Light-Induced Kinetic Effects on atoms, ions and molecules, Proceedings of the LIKE workshop, edited by L. Moi (E.T.S., Pisa), 1991.

[14] K. I. Petsas, Thèse de l'université Pierre et Marie Curie, Paris (1996).

[15] P. Reimann, M. Grifoni and P. Hänggi, Phys. Rev. Lett. 79, 10 (1997). 